\title{
MENINGEOMAS. ACOMETIMENTO ISOLADO DE NERVOS CRANIANOS
}

\author{
OSWaldo Ricciardi CRUZ * \\ Rolando Tenuto **
}

A sindrome paralitica unilateral de todos os nervos cranianos - síndrome de Guillain, Alajouanine e Garcin - sugere a existência de processo expansivo na base do crânio. As principais caracteristicas dessa sindrome são o acometimento unilateral e isolado de todos ou da maioria dos nervos cranianos, não havendo ou sendo tardia a ocorrência de hipertensão intracraniana. As neoplasias intracranianas de crescimento lento, particularmente os meningeomas, a despeito do grande volume que possam assumir, determinam, em muitos casos, apenas acometimento de nervos cranianos, sem provocar hipertensão intracraniana. A integridade das funções cerebrais, a ausência de distúrbios sensitivo-motores, assim como a normalidade do exame oftalmoscópico ou o aparecimento tardio de edema de papila são ressaltados em observações de André Thomas ${ }^{3}$. Montanaro e col. ${ }^{7}$ admitem que a extensão lenta e progressiva ou a infiltração precoce das meninges pelo tumor são responsáveis pelas lesões unilaterais dos nervos cranianos.

O acometimento unilateral e isolado dos V e VII nervos cranianos em casos de meningeomas supratentoriais constitui raridade, tendo sido registrado por alguns autores que salientam seu falso valor localizatório. David $^{4}$ foi o primeiro a chamar a atenção para o acometimento do trigêmeo em casos de meningeomas parassagitais, lembrando a dificuldade do diagnóstico topográfico quando se toma por base êsse sinal de localização. Recentemente Alajouanine e col.1,2 se referiram às mesmas dificuldades em casos de meningeomas frontais e parassagitais em que ocorrem sintomas de comprometimento isolado de nervos cranianos. Um dos mecanismos aventados por êsses autores para explicar tais acometimentos em processos situados à distância é dinâmico, decorrendo da hipertensão intracraniana que determina o estiramento dos nervos no trajeto entre sua emergência aparente do tronco cerebral e sua saída pelos orifícios da base do crânio; a pressão se exerce como fôrça dirigida do alto para baixo e da frente para trás, determinando o estiramento e conseqüente lesão dos nervos que mantêm rela-

Trabalho da Clinica Neurológica da Faculdade de Medicina da Universidade de São Paulo (Prof. Adherbal Tolosa). *Assistente de Neurocirurgia; ** Chefe do Serviço de Neurocirurgia. 
ções topográficas com a pirâmide petrosa, particularmente o nervo trigêmeo. Os meningeomas da fossa posterior, segundo Cushing e Eisenhardt ${ }^{5}$ e Marklan e col. ${ }^{6}$, determinam, com maior freqüência, sinais de comprometimento de nervos cranianos por meio da compressão direta exercida pela massa neoplásica.

\section{MATERIAL}

Nosso material consta de 41 casos de meningeomas intracranianos com confirmação neurocirúrgica e anátomo-patológica. A revisão que fizemos visou verificar a incidência do acometimento isolado de nervos cranianos e seu valor como elemento localizatório; colateralmente procuramos verificar a freqüência com que êsses tumores ocasionam hipertensão intracraniana.

Em 30:41 casos o exame oftalmológico revelou edema de papila uni ou bilateral; em 10:41 casos não havia edema de papila; em um caso o exame não foi realizado. Em 11:41 casos a pressão do líqüido cefalorraquidiano colhido por via suboccipital e com o paciente em decúbito lateral, era igual ou ligeiramente inferior a $20 \mathrm{~cm}$ de água (manômetro de Claude); em 21:41 casos a pressão do liqüido cefalorraquidiano foi superior a $20 \mathrm{~cm}$ de água, sendo que em dois casos a colheita se fêz por via ventricular; em 9 casos não foi feito o exame do liquiido cefalorraquidiano. O confronto entre êsses dados - exame oftalmológico e manometria do liqüido cefalorraquidiano - mostra que em 6 casos dos 11 sem hipertensão liquórica o exame oftalmológico revelou a existência de edema de papila e que, por outro lado, em 4 dos 21 casos com hipertensão liquórica o exame de fundo de ôlho era normal.

O acometimento de nervos cranianos, isolado ou associado a outros sinais neurológicos está registrado no quadro 1 . Em 25:41 casos o processo expansivo determinou distúrbios motores, sensitivos ou de coordenação por comprometimento do parênquima nervoso, sendo que em apenas 10 dêsses (casos $1,5,9,10,12,15,19$, 23,25 e 28) havia sinais de acometimento de nervos cranianos. O nervo motor ocular comum foi comprometido em 4 (casos 1, 9, 10 e 23); o abducente em 2 (casos 12 e 28); o acústico no caso 15; o glossofaríngeo no caso 25; o trigêmeo no caso 5; os nervos trigêmeo, facial, glossofaríngeo, vago e acessório espinhal no caso 19. Em 14:41 (casos $2,6,7,11,16,17,22,27,33,34,35,37,38$ e 40) não havia qualquer sinal neurológico que indicasse lesão do parênquima nervoso. Em 2:41 (casos 32 e 41) foi observado acometimento unilateral e isolado de nervos cranianos: os nervos abducentes e acústico estavam acometidos no caso 32; os nervos motor ocular comum, abducente, troclear, facial e acústico foram comprometidos no caso 41.

Assim, êste estudo mostra que: a) em 14:41 casos de meningeomas não havia qualquer sinal neurológico indicativo da sede do processo expansivo; b) em 25:41 casos os sinais localizatórios permitiam orientar clinicamente o diagnóstico; c) em 2:41 casos os elementos clínicos encontrados - acometimento de nervos cranianos - induziam a falsa localização da neoplasia. A seguir, relataremos mais detalhadamente êstes dois últimos casos.

Caso 32 - Y.S.M., com 46 anos de idade, sexo feminino, branca, internada em 25-2-1959 (Reg. 546.179). Há um ano cefaléia intensa, intermitente, de intensidade progressiva. Há um mês perda acentuada da acuidade visual. Exame clínico normal. Exame neurológico: edema de papila bilateral; paresia do abducente e hipoacusia à direita. 


\begin{tabular}{|c|c|c|c|c|c|}
\hline Casos & Reg. & $\begin{array}{c}\text { Paralisias de nervos } \\
\text { cranianos }\end{array}$ & $\begin{array}{l}\text { Outros distúrbios } \\
\text { neurológicos }\end{array}$ & $\begin{array}{l}\text { Localização da } \\
\quad \text { Lado }\end{array}$ & $\begin{array}{l}\text { neoplasia } \\
\text { Sede }\end{array}$ \\
\hline 1 & 294983 & III D & Hemiparesia $\mathrm{E}$ & $\mathrm{D}$ & $P$ \\
\hline 2 & 057410 & - & & $\mathrm{E}$ & $\mathrm{F}$ \\
\hline 3 & 331044 & - & Afasia & $\mathrm{E}$ & $\mathrm{F}$ \\
\hline 4 & 389787 & - & Hemiparesia $\mathrm{E}$ & $\mathrm{D}$ & $F$ \\
\hline 5 & 425676 & V D & Hemiplegia D & $\mathbf{E}$ & Pps \\
\hline 6 & 377951 & - & - & $\mathrm{D}$ & $\mathrm{T}$ \\
\hline 7 & 312346 & - & - & $\mathrm{E}$ & $\mathrm{F}$ \\
\hline 8 & 312468 & - & Hemiparesia $\mathrm{E}$ & $\mathrm{D}$ & $P$ \\
\hline 9 & 350195 & III, IV, VI D & & D & FT \\
\hline 10 & 151935 & III $\mathrm{D}$ & Hemiplegia $\mathrm{E}$ & $\mathrm{D}$ & $\mathbf{T}$ \\
\hline 11 & 332130 & - & - & $\mathrm{D}$ & $\mathrm{T}$ \\
\hline 12 & 041954 & VI $\mathrm{E}$ & Paraplegia crural & M & $\mathrm{Ba}^{*}$ \\
\hline 13 & 258335 & - & Hemiparesia D & $\mathrm{E}$ & $\mathrm{P}$ \\
\hline 14 & 338288 & - & Hemiparesia $\mathrm{E}$ & $\mathrm{D}$ & $\mathrm{FP}$ \\
\hline 15 & 124718 & VIII D & Incoordenação & $\mathrm{Bi}$ & $F$ \\
\hline 16 & 146349 & - & - & $\mathrm{E}$ & $\mathrm{T}$ \\
\hline 17 & 339982 & - & - & $\mathrm{E}$ & $\mathrm{T}$ \\
\hline 18 & 420651 & - & Hemiplegia $\mathrm{E}$ & $\mathrm{D}$ & PT \\
\hline 19 & 047785 & V, VIII D & & & $\mathrm{BA}$ \\
\hline & & VII, IX, X, XII E & Hemiplegia $\mathrm{E}$ & M & $\mathrm{O}$ \\
\hline 20 & 271159 & - & Hemiplegia $\mathrm{E}$ & $\mathrm{D}$ & Fps \\
\hline 21 & 426992 & - & Paraparesia crural & $\mathrm{D}$ & $\mathrm{F}$ \\
\hline 22 & 427533 & - & - & $\mathrm{E}$ & Pps \\
\hline 23 & 430767 & III $\mathrm{D}$ & Hemiplegia $\mathrm{D}$ & $\mathrm{E}$ & Pps \\
\hline 24 & 463643 & - & Hemiplegia $E$ & $\mathrm{D}$ & It \\
\hline 25 & 467075 & IX E & Incoordenação & $\mathrm{M}$ & $\mathrm{T}$ \\
\hline 26 & 484906 & - & Incoordenação & $\mathrm{D}$ & $\mathrm{F}$ \\
\hline 27 & 493562 & - & - & $\mathrm{D}$ & $\mathbf{F}$ \\
\hline 28 & 481723 & VI D & Monoparesia D & $\mathrm{E}$ & $\mathrm{F}$ \\
\hline 29 & 488496 & - & $\begin{array}{l}\text { Rigidez de decorti- } \\
\text { cação }\end{array}$ & $\mathrm{E}$ & It \\
\hline 30 & 478780 & - & Incordenação & $\mathrm{E}$ & It \\
\hline 31 & 528080 & 一 & Hemiparesia $\mathrm{E}$ & $\mathrm{D}$ & FT \\
\hline 32 & 546179 & VI, VIII D & - & $\mathrm{D}$ & $\mathrm{T}$ \\
\hline 33 & 366890 & - & - & $\mathrm{D}$ & $\mathbf{F}$ \\
\hline 34 & 323066 & - & - & $\mathrm{Bi}$ & Pps \\
\hline 35 & 221801 & - & - & $\mathrm{E}$ & $\mathbf{F}$ \\
\hline 36 & 234320 & - & Hipoestesia $\mathrm{E}$ & M & $\mathrm{F}$ \\
\hline 37 & 434193 & - & - & $\mathrm{D}$ & $\mathbf{T}$ \\
\hline 38 & 424281 & - & - & $\mathrm{E}$ & $\mathrm{T}$ \\
\hline 39 & 212421 & - & Hemiplegia $\mathrm{E}$ & $\mathrm{D}$ & $\mathrm{P}$ \\
\hline 40 & 397905 & - & - & $\mathrm{D}$ & $\mathbf{F}$ \\
\hline 41 & 464700 & $\begin{array}{l}\text { III, IV, V, VI, } \\
\text { VII, VIII E }\end{array}$ & 一 & M & $\mathrm{Ba}$ \\
\hline
\end{tabular}

Quadro 1 - Resumo da sintomatologia em 41 casos de meningeomas intracranianos. Legenda: D, direito; E, esquerdo; F, frontal; $\mathrm{P}$, parietal; $\mathrm{T}$, temporal; $\mathrm{O}$, occipital; $M$, medial; $\mathrm{Ba}$, base do crânio; ps, parassagital; It, infratentorial; $\mathrm{Bi}$, bilateral. * No caso 12 havia, concomitantemente à neoplasia intracraniana, processo compressivo medular. 
Exames subsidiários - Electrencefalograma: sinais de sofrimento cerebral no hemisfério direito. Exame radiológico do crânio: alargamento do assoalho da sela túrcica e erosão das apófises clinóides posteriores. Exame do liqüido cefalorraquidiano, em punção suboccipital e decúbito lateral: líqüido límpido e incolor, pressão inicial $80 \mathrm{~cm}$ de água (manômetro de Claude), pressão final $20 \mathrm{~cm}$; $120 \mathrm{mg}$ de proteínas por $100 \mathrm{ml}$; reaçōes de Pandy e Nonne positivas; reações coloidais com desvio para a esquerda. Angiografia cerebral via artéria carótida direita: quadro angiográfico de processo expansivo vascularizado na região temporal direita (fig. 1).
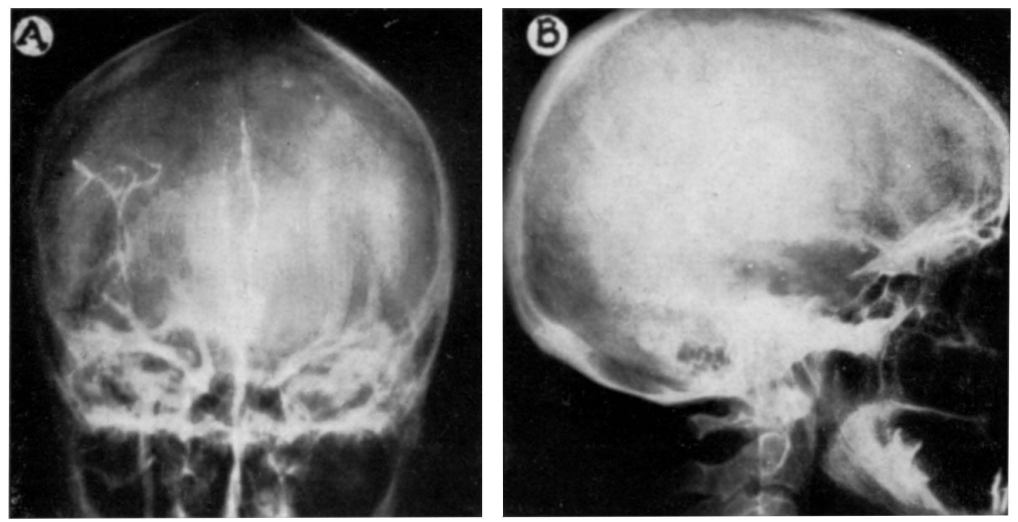

Fig. 1 - Caso 32 (Y.S.M.). Processo neoplásico temporal: em A, angiografia cerebral via artéria carótida direita, mostrando, no tempo arterial e em incidência sagital, o desvio da artéria cerebral anterior para a esquerda; em $B$, tempo venoso em incidência lateral, mostrando a retenção de contraste em forma nodular.

Intervenção cirürgica e evolução - Após craniotomia frontotemporal direita foi encontrado tumor temporal vascularizado, aderente à dura mater e fàcilmente enucleável. Biopsia: Meningeoma fibromatoso. Pós-operatório sem anormalidades, tendo a paciente recebido alta, sem distúrbios neurológico algum, em 15-3-59.

CASo 41 - M.A.A., com 12 anos de idade, sexo feminino, branca, internada em 16-1-1959 (Reg. 464700). Há très anos estrabismo interno no ôlho esquerdo, tendo sido feita, há dois anos, operação para correção dêsse estrabismo. Cinco meses após a operação ocorreu paralisia de todos os músculos extrínsecos do globo ocular esquerdo e, a seguir, protusão dêsse ôlho; concomitantemente a paciente acusou diminuição da acuidade visual, tendo o exame oftalmoscópico revelado edema de papila no ôlho esquerdo. A paciente estêve internada na Clinica oftalmológica, onde o exame radiológico do crânio mostrou sinais de extensa neoplasia que ocupava grande parte da fossa média esquerda e o fundo da órbita esquerda. A paciente foi encaminhada, então, para a Clínica Neurológica, onde, além da oftalmoplegia completa à esquerda e edema papilar, foi assinalada a existência de paralisia do ramo motor do nervo trigêmeo, paralisia facial periférica e hipoacusia unilaterais à esquerda.

Exames subsidiários - Electrencefalograma: anormalidade fronto-temporal à direita. Exame do líqüido cefalorraquidiano em punção suboccipital e decúbito la- 
teral: liqüido limpido e incolor; pressão inicial $33 \mathrm{~cm}$ de água (manômetro de Claude), pressão final $19 \mathrm{~cm} ; 40 \mathrm{mg}$ de proteinas por $100 \mathrm{ml}$. Angiografia cerebral bilateral via artéria carótida interna: quadro angiográfico de processo expansivo da base do crânio predominando na fossa média esquerda (figs. 1 e 2).
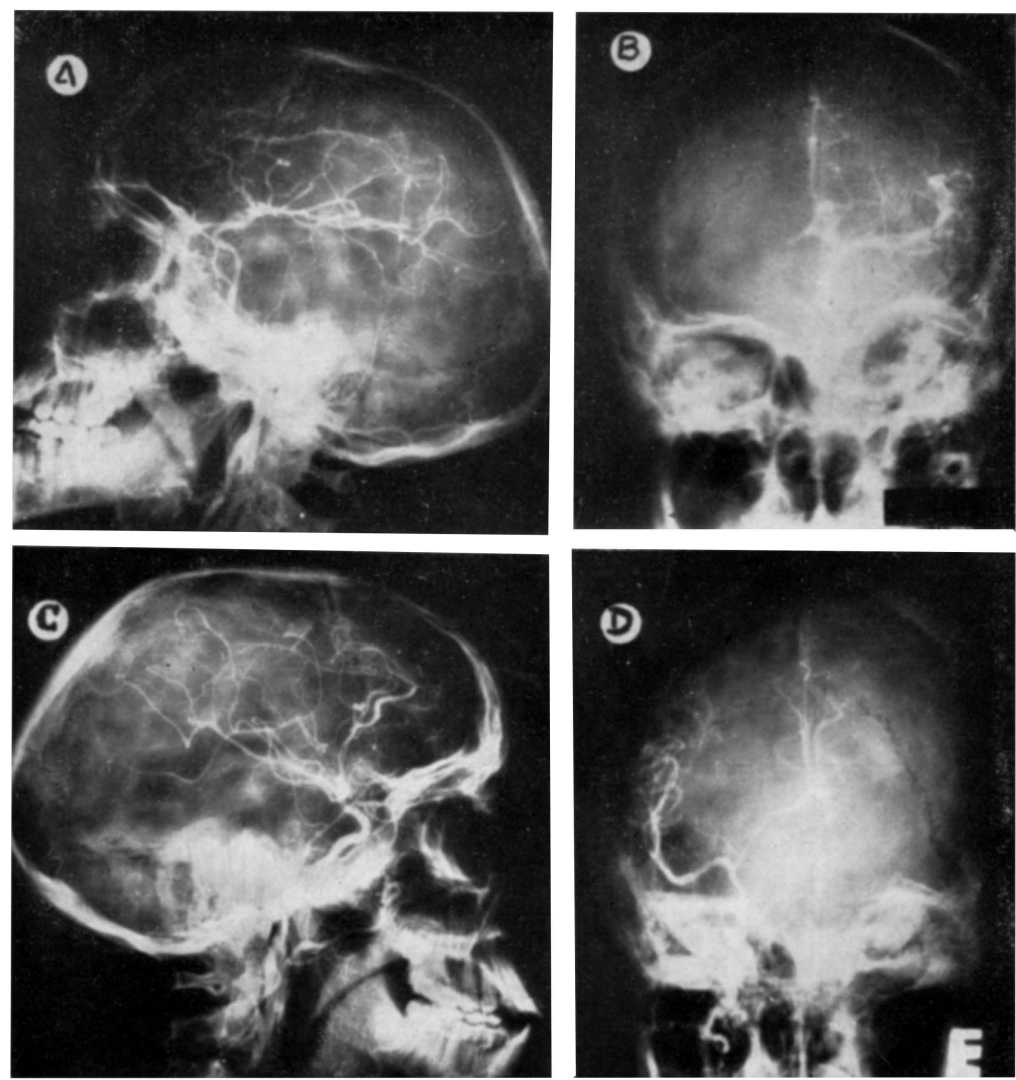

Fig. 2 - Caso 41 (M.A.A.). Processo neoplásico na base do crânio: em $A$ e $B$, angiografia cerebral via artéria carótida esquerda nas incidências lateral e sagital, mostrando acentuado desvio da artéria cerebral média, permanecendo a artéria cerebral anterior no plano médio sagital; em $C$ e $D$, angiografia cerebral via carótida direita. mostrando o desvio para cima e para a direita da porçäo horizontal (segmento $A_{1}$ ) da artéria cerebral anterior.

Intervenção cirúrgica, evoluçũo, necropsia - Com o diagnóstico de tumor da base do crânio predominando na fossa média esquerda, foi feita craniotomia frontotêmporo-parietal esquerda, sendo extirpado parcialmente extensa neoplasia que ocupava tôda a fossa média e invadia a fossa anterior. Durante o ato cirúrgico ocorreu edema cerebral agudo. Biopsia: Meningeoma meningotelial. Pós-operatório imediato acidentado, entrando a paciente em coma profundo; no terceiro dia ocorreu 
broncopneumonia que determinou o óbito. Necropsia - No encéfalo, massa tumoral que ocupava tôda a extensão da base do crânio, invadindo as fossas média, anterior e posterior. Diagnóstico histológico - Meningeoma meningotelial.

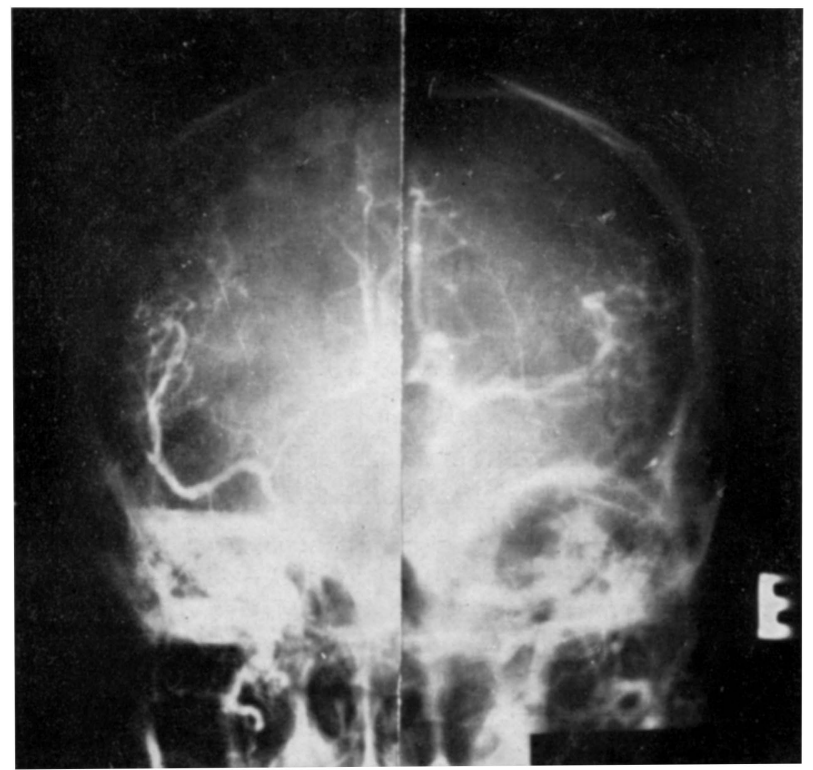

Fig. 3 Caso 41 (M.A.A.). Montagem das angiografias exibidas na figura 2 , obtidas mediante injeção de contraste à direita e à esquerda em incidência ântero-posterior, demonstrando a grande extensĩo do tumor na base do crânio.

\section{COMENTÁRIOS}

Os sinais neurológicos observados na maioria dos casos de meningeomas - e nesta série de 25:41 casos — são suficientes para o diagnóstico clínico e topográfico. Em alguns casos (meningeomas osteoblásticos ${ }^{5}$ ), a localização e o caráter histológico do tumor, a despeito da não existência de sinais neurológicos, permitem, pela palpação do segmento cefálico, o reconhecimento topográfico; assim, no caso 9 , uma tumoração temporal dura e fixa aos planos profundos permitiu o diagnóstico da neoplasia.

Em outros casos - como ocorreu em 14:41 dos casos desta série - acomodando-se a hidrodinâmica intracraniana ao crescimento lento da neoplasia, não ocorrem sinais neurológicos de localização, o que dificulta o diagnóstico clínico e topográfico.

As dificuldades para o diagnóstico topográfico aumentam quando aparecem sinais indicativos de lesões neurológicas ocorrendo à distância da sede real da neoplasia. Em casos de meningeomas supratentoriais pode haver acometimento isolado e unilateral de nervos cranianos, determinando o 
aparecimento de falsos sinais de localização. A despeito da pobreza dos dados clínico-neurológicos, o exame radiológico do crânio e outros exames especializados permitem, em alguns casos, o diagnóstico topográfico.

Em dois casos desta série (casos 32 e 41) o único indício de lesão do sistema nervoso era constituido por acometimento isolado e unilateral de nervos cranianos, como ocorreu nos casos relatados por David ${ }^{4}$, André Tho$\operatorname{mas}^{3}$ e Alajouanine e col.1, ${ }^{2}$.

No caso 32 (meningeoma temporal), à paralisia do nervo abducente não foi dado valor localizatório, pois a pressão do líqüido cefalorraquidiano era de $80 \mathrm{~cm}$ de água, sendo sabido que êsse nervo é freqüentemente comprometido quando há hipertensão intracraniana. Neste caso havia também acometimento unilateral do nervo acústico que conduziria a diagnóstico topográfico errôneo, pois sugeria estar a neoplasia situada na fossa craniana posterior.

No caso 41 (meningeoma na base do crânio), a orientação terapêutica inicial seguida em outra clínica visou o acometimento isolado de um nervo craniano (abducente); o acometimento ulterior de outros nervos cranianos e o aparecimento de edema de papila levaram à prática de exames subsidiários que conduziram corretamente o diagnóstico topográfico do processo neoplásico. Êsse caso, muito semelhante àquele revelado por André Thomas ${ }^{3}$ - meningeoma da fossa posterior com paralisia de seis nervos cranianos - vem confirmar nossa observação quanto às dificuldades e os riscos do diagnóstico clínico sem documentação complementar; a despeito do grande volume da neoplasia, a afecção evoluiu sem sinais de comprometimento do sistema nervoso central, só se manifestando por acometimento lento e progressivo de nervos cranianos.

\section{RESUMO}

Os autores fazem uma revisão de 41 casos de meningeomas intracranianos, chamando a atenção para a raridade dos acometimentos isolados $e$ unilaterais dos nervos cranianos, para ressaltar o falso valor localizatório dêsses acometimentos em alguns casos. Em um de seus casos (caso 41), seis nervos cranianos foram comprometidos sem serem surpreendidos outros sinais que indicassem lesōes das diferentes funções cerebrais. Em outro (caso 32), de meningeoma localizado no lobo temporal, o comprometimento isolado de VI e VIII nervos cranianos poderia ter induzido ao diagnóstico de um processo localizado na fossa posterior.

\section{SUMMARY}

Meningeomas. Involvement of cranial nerves as the only presenting sign.

A review of 41 cases of intracranial meningeomas, some with unilateral isolated lesions of cranial nerves, is presented. From these 41 cases, 25 
presented neurological symptoms and 14 were asymptomatic, the diagnosis being made only by subsidiary methods. In two cases of supra-tentorial meningeomas there were only signs of involvement of cranial nerves. In one of them (case 41) six cranial nerves were affected without any other signs of lesion of the nervous system. In other case (case 32 - temporal meningeoma) involvement of the VIth and VIII ${ }^{\text {th }}$ nerves was the only presenting sign, thus suggesting a posterior fossa localization.

\section{REFERENCIAS}

1. AlajouAnine, Th.; CASTAIGNE, P.; NICK, J.; PERTuISET, B.; BARBIZET, J.; NAMIN, P. - L'atteinte du trijumeau et du facial dans les méningeomes frontaux sagittaux et parasagittaux. Rev. Neurol., 88:225, 1953. 2. ALAJOUANINE, Th.; CELICE, J.; CASTIGNE, P.; PERTUISET, B. - Sur les faux symptômes de localisation dans les tumeurs cérébrales (méningeome frontal avec paralysie des nerfs craniens Ve., VIe., VIIe, paires). Rev. Neurol., 85:382, 1951.3 . THOMAS, A. - Paralysie unilaterale des six derniers nerfs craniens: méningeome de la fosse cérébelleuse. Prèsse Med., 45:1785 (dezembro, 11), 1937. 4. DAVID, M. - De l'atteinte unilaterale et homolaterale du trijumeau au cours de certains méningeomes parasagittaux. Rev. Neurol., 73:11, 1941. 5. CUSHING, H.; EISENHARDT, L. Meningeomas. Charles C. Thomas, Baltimore, 1938. 6. MARKHAN, J. W.; FAGER, C.; HORRAX, G.; POPPEN, J. L. - Meningeomes of the posterior fossa. Arch. Neurol. a. Psychiat., 74:163, 1955. 7. MONTANARO, J. C.; FRANCHINI, Y.; BLOTTA, F. - Sindrome paralítico unilateral global de los nervios cranianos (de Guillain, Alajouanine y Garcin) por neoplasia basilar. Semana Med, (Buenos Aires), 50:1118 (novembro, 11), 1953.

Clinica Neurológica. Hospital das Clínicas da Fac. Med. da Univ. de São Paulo - Caixa Postal 3461 - São Paulo, Brasil. 